Pacific Journal of Mathematics

ON THE ABSOLUTE HAUSDORFF SUMMABILITY OF A 


\title{
ON THE ABSOLUTE HAUSDORFF SUMMABILITY OF A FOURIER SERIES
}

\author{
S. N. LAL AND SIYA RAM
}

\begin{abstract}
In this paper a theorem on the absolute Hausdorff summability of a series associated with a Fourier series has been established. This theorem unifies and extends various known results.
\end{abstract}

1. Let $\mu_{n}$ be a sequence of real or complex numbers and write $\Delta^{0} \mu_{n}=\mu_{n}, \Delta^{p} \mu_{n}=\Delta^{p-1} \mu_{n}-\Delta^{p-1} \mu_{n+1}, \quad p \geqq 1$.

If $S_{n}$ denotes the sequence of partial sums of the series $\sum_{n=0}^{\infty} a_{n}$, the transformation

$$
t_{m}=\sum_{n=0}^{m}\left(\begin{array}{c}
m \\
n
\end{array}\right)\left(\Delta^{m-n} \mu_{n}\right) S_{n}
$$

defines the sequence $\left\{t_{m}\right\}$ of $(H, \mu)$ means or the Hausdorff means $[3,12]$ of the sequence $\left\{S_{n}\right\}$. The series $\sum a_{n}$ is said to be summable $(H, \mu)$ to the sum $s$ if $\lim _{m \rightarrow \infty} t_{m}=s$ and is said to be absolutely summable $(H, \iota)$ or summable $|H, \mu|$ if

$$
\sum_{n=1}^{\infty}\left|t_{n}-t_{n-1}\right|<C^{1}
$$

In order that $(H, \iota)$ should be a convergence preserving transformation it is necessary and sufficient that $\mu_{n}$ should be a moment constant, that is, there exists a function $\chi(x)$ of bounded variation in $0 \leqq x \leqq 1$, such that

$$
\mu_{n}=\int_{0}^{1} x^{n} d \chi(x), \quad n=0,1,2, \cdots .
$$

We may suppose without loss of generality that $\chi(0)=0$. If also, $\chi(1)=1$ and $\chi(+0)=\chi(0)=0$, so that $\chi(x)$ is continuous at the origin, then $\ell_{n}$ is a regular moment constant and $(H, \mu)$ is a regular Hausdorff transformation [3]. It is known that the sequence to sequence Hausdorff transformation is absolute convergence preserving or absolutely regular if and only if it is a convergence preserving or regular transformation of the same type [4, 8, 9].

In the case in which

$$
\chi(x)=1-(1-x)^{\delta}, \quad \delta>0,
$$

1 Throughout the paper $C$ denotes a positive constant not necessarily the same at each occurrence. 
the method $(H, \mu)$ reduces to the well known Cesàro method $(C, \delta)[3$, 12].

2. Let $f(t)$ be a periodic function with period $2 \pi$ and integrable in the Lebesgue sense in $(-\pi, \pi)$. Let the Fourier series of $f(t)$ be

$$
\sum_{n=1}^{\infty}\left(a_{n} \operatorname{cosn} t+b_{n} \sin n t\right) \equiv \sum_{n=1}^{\infty} A_{n}(t),
$$

it being assumed that the constant term is zero.

The $\varepsilon$ th forward and backward fractional integrals of a function $g(x)$, which is Lebesgue integrable in $(0,1)$, are respectively defined as

$$
g_{\varepsilon}^{+}(x)=\frac{1}{\Gamma(\varepsilon)} \int_{0}^{x}(x-u)^{\varepsilon-1} g(u) d u
$$

and

$$
g_{\varepsilon}^{-}(x)=\frac{1}{\Gamma(\varepsilon)} \int_{x}^{1}(u-x)^{\varepsilon-1} g(u) d u .
$$

These integrals exist almost everywhere for $\varepsilon>0$.

We write

$$
\begin{aligned}
\phi(t) & =\frac{1}{2}\{f(x+t)+f(x-t)\} ; \\
\Phi_{0}(t) & =\phi(t) ; \\
\Phi_{\alpha}(t) & =\frac{1}{\Gamma(\alpha)} \int_{0}^{t}(t-u)^{\alpha-1} \phi(u) d u, \quad \alpha>0 ; \\
\phi_{\alpha}(t) & =\Gamma(\alpha+1) t^{-\alpha} \Phi_{\alpha}(t), \quad \alpha \geqq 0 ; \\
M(n, x, t) & =\sum_{\nu=1}^{n} \nu\left(\begin{array}{l}
n \\
\nu
\end{array}\right) \varepsilon(\nu) x^{\nu}(1-x)^{n-\nu} \cos \nu t ; \\
N(n, x, t) & =\sum_{\nu=1}^{n}\left(\begin{array}{l}
n \\
\nu
\end{array}\right) \varepsilon(\nu) x^{\nu}(1-x)^{n-\nu} \sin \nu t ; \\
L(\chi ; n, t) & =\frac{2}{\pi} \sum_{\nu=1}^{n}\left(\begin{array}{l}
n \\
\nu
\end{array}\right) \varepsilon(\nu) \sin \nu t \int_{0}^{1} x^{\nu}(1-x)^{n-\nu} d \chi(x) ; \\
I(\chi ; n, u) & =\frac{1}{\Gamma(1-\alpha)} \int_{u}^{-}(t-u)^{-\alpha} \frac{d}{d t} L(\chi ; n, t) d t ; \\
J(\chi ; n, u) & =\frac{1}{\Gamma(1+\alpha)} \int_{0}^{u} v^{\alpha} \frac{d}{d v} I(\chi ; n, v) d v .
\end{aligned}
$$

3. In this paper we establish the following:

TheOREM. Let $\varepsilon(t)$ be a positive and monotonic nondecreasing 
function of $t$ such that

$$
\sum_{n=[1 / t]+1}^{\infty} \frac{\varepsilon(n)}{n^{1+\gamma-\alpha} t^{\gamma-\alpha}}=O\left(\varepsilon\left(\frac{k}{t}\right)\right), \quad(0<t<\pi, k>\pi)
$$

and

$$
\int_{0}^{\pi} \varepsilon\left(\frac{k}{t}\right)\left|d \phi_{\alpha}(t)\right|<C
$$

If

$$
\begin{gathered}
\text { either (a) } \chi(u)=g_{1+\gamma}^{+}(u)+C, \quad(0 \leqq \alpha<\gamma<1) \\
\text { or } \quad \text { (b) } \chi(u)=g_{1+\gamma}^{-}(u)+C,
\end{gathered}
$$

for some function $g(u)$ which is Lebesgue integrable in $(0,1)$, then the series $\sum \varepsilon(n) A_{n}(t)$ is summable $|H, \mu|$ at the point $t=x$, it being assumed that the transformation $(H, \mu)$ is convergence preserving.

Taking $\varepsilon(t)=1$ and $\alpha=0$ the above theorem reduces to a recent result on the absolute Hausdorff summability of a Fourier series ([11], Theorem 1) which in turn includes ${ }^{2}$ a result of Bosanquet ([1], Theorem 1) on the absolute Cesàro summability of a Fourier series and the case $0<\alpha<1$ covers another result on the absolute Cesàro summability of a Fourier series ([2], Theorem 1). Also for $\alpha=0$ choosing $\varepsilon(t)=t^{\beta}$ and $\gamma=\beta+\delta(\beta>0, \delta>0)$ we get another result ([10], Theorem 1) on the absolute Hausdorff summability which is known to include a theorem on the absolute Cesàro summability of the series $\sum n^{\beta} A_{n}(t)$ due to Mohanty ([6], Theorem 1). Further choosing $\varepsilon(t)=\log (1+t)$ we get (cf. [7])

Theorem A. If

$$
\int_{0}^{\pi} \log \frac{k}{t}\left|d \phi_{\alpha}(t)\right|<C \quad(k>\pi)
$$

and

$$
\begin{array}{cl}
\text { either (a) } \quad \chi(u)=g_{1+\gamma}^{+}(u)+C, \quad(0 \leqq \alpha<\gamma<1) \\
\text { or } \quad \text { (b) } \chi(u)=g_{1+\gamma}^{-}(u)+C,
\end{array}
$$

for some function $g(u)$ which is Lebesgue integrable in $(0,1)$, then the series $\sum \log (n+1) A_{n}(t)$ is summable $|H, \mu|$, at the point $t=x$, it being assumed that the transformation $(H, \mu)$ is convergence preserving.

4. We require the following lemmas for the proof of our theorem.

${ }^{2}$ While this paper was in press, a paper due to B. Kuttner and N. Tripathi (Quart. J. Math., 22 (1971), 229-308) appeared in which it is shown that Tripathi's theorem can be deduced from the result of Bosanquet. 
Lemma 1 [4]. Let $\left\{t_{n}\right\}$ and $\left\{S_{n}\right\}$ be the partial sums of the series $\sum b_{n}$ and $\sum a_{n}$ respectively. Then the sequence to sequence transformation

$$
t_{m}=\sum_{n=0}^{m}\left(\begin{array}{c}
m \\
n
\end{array}\right)\left(\Delta^{m-n} \mu_{n}\right) S_{n}
$$

can be put in the series to series form as

$$
\begin{aligned}
b_{m} & =\frac{1}{m} \sum_{n=1}^{m}\left(\begin{array}{c}
m \\
n
\end{array}\right)\left(\Delta^{m-n} \mu_{n}\right) n a_{n}, \\
b_{0} & =a_{0} .
\end{aligned}
$$

Lemma 2 [5]. If $g(x)$ and $h(x)$ are Lebesgue integrable in $(0,1)$, then for $\varepsilon>0$

$$
\int_{0}^{1} g_{\varepsilon}^{+}(x) h(x) d x=\int_{0}^{1} g(x) h_{\varepsilon}^{-}(x) d x
$$

Lemma 3.

$$
I_{1} \equiv \int_{0}^{x} N(n, 1-v, t) d v=O\left(\frac{\varepsilon(n)}{n t}\right)
$$

and

$$
I_{2} \equiv \int_{0}^{x} M(n, 1-v, t) d v=O\left(\frac{\varepsilon(n)}{t}\right)
$$

uniformly for $x$ in $(0,1)$.

Proof. By Abel's transformation, we have

$$
\begin{aligned}
I_{1}= & \int_{0}^{x}\left(\sum_{\nu=1}^{n}\left(\begin{array}{l}
n \\
\nu
\end{array}\right) \varepsilon(\nu)(1-\nu)^{\nu} v^{n-\nu} \sin \nu t\right) d v \\
= & \int_{0}^{x}\left[\sum_{\nu=1}^{n-1} \Delta_{\nu}\left\{\left(\begin{array}{l}
n \\
\nu
\end{array}\right) \varepsilon(\nu)(1-v)^{\nu} v^{n-\nu}\right\} \sum_{r=1}^{\nu} \sin r t\right. \\
& \left.+\left(\begin{array}{l}
n \\
n
\end{array}\right) \varepsilon(n)(1-v)^{n} \sum_{\nu=1}^{n} \sin \nu t\right] d v \\
= & O\left(\frac{1}{t}\right) \sum_{\nu=1}^{n-1}\left|\Delta_{\nu}\left(\varepsilon(\nu) p_{\nu}(x)\right)\right|+O\left(\frac{\varepsilon(n)}{n t}\right),
\end{aligned}
$$

where

$$
p_{\nu}(x)=\left(\begin{array}{l}
n \\
\nu
\end{array}\right) \int_{0}^{x} v^{n-\nu}(1-v)^{\nu} d v, \quad(1 \leqq \nu \leqq n-1) .
$$

We observe that $\varepsilon(\nu) p_{\nu}(x)(1 \leqq \nu \leqq n-1)$ is a nondecreasing function of $\nu$ for fixed $x$, since by hypothesis $\varepsilon(\nu)$ is non-decreasing and 


$$
\begin{aligned}
p_{\nu}(x) & =\left(\begin{array}{l}
n \\
\nu
\end{array}\right) \int_{0}^{x} v^{n-\nu}(1-v)^{\nu} d v \\
& =\left(\begin{array}{l}
n \\
\nu
\end{array}\right)\left[-\frac{v^{n-\nu}(1-v)^{\nu+1}}{\nu+1}\right]_{0}^{x}+\left(\begin{array}{l}
n \\
\nu
\end{array}\right) \frac{(n-\nu)}{(\nu-1)} \int_{0}^{x} v^{n-\nu-1}(1-v)^{\nu+1} d v \\
& =\left(\begin{array}{l}
n \\
\nu+1
\end{array}\right) \int_{0}^{x} v^{n-\nu-1}(1-v)^{\nu+1} d v-\frac{1}{(\nu+1)}\left(\begin{array}{l}
n \\
\nu
\end{array}\right) x^{n-\nu}(1-x)^{\nu+1} \\
& =p_{\nu+1}(x)-\frac{1}{(\nu+1)}\left(\begin{array}{c}
n \\
\nu
\end{array}\right) x^{n-\nu}(1-x)^{\nu+1} \\
& <p_{\nu+1}(x) .
\end{aligned}
$$

Hence

$$
\begin{aligned}
I_{1} & =O\left(\frac{1}{t}\right) \sum_{\nu=1}^{n-1}\left[\varepsilon(\nu+1) p_{\nu+1}(x)-\varepsilon(\nu) p_{\nu}(x)\right]+O\left(\frac{\varepsilon(n)}{n t}\right) \\
& =O\left(\frac{\varepsilon(n)}{t}\right) \int_{0}^{x}(1-v)^{n} d v+O\left(\frac{\varepsilon(n)}{n t}\right) \\
& =O\left(\frac{\varepsilon(n)}{n t}\right) .
\end{aligned}
$$

$I_{2}$ can be similarly estimated. Hence the lemma.

LeMma 4. For $0<\gamma<1$,

$$
J_{1} \equiv \int_{0}^{1-x}(1-x-u)^{\gamma-1} N(n, 1-u, t) d u=O\left(\frac{\varepsilon(n)}{n^{\gamma} t^{\gamma}}\right)
$$

and

$$
J_{2} \equiv \int_{0}^{1-x}(1-x-u)^{\gamma-1} M(n, 1-u, t) d u=O\left(\frac{\varepsilon(n)}{n^{\gamma-1} t^{r}}\right)
$$

uniformly for $x$ in $(0,1)$.

\section{Proof. Since}

$$
\begin{aligned}
|N(n, 1-u, t)| & \leqq \varepsilon(n) \sum_{\nu=1}^{n}\left(\begin{array}{l}
n \\
\nu
\end{array}\right)(1-u)^{\nu} u^{n-\nu} \\
& \leqq \varepsilon(n)
\end{aligned}
$$

we have

$$
\begin{aligned}
J_{1} & =\int_{0}^{1-x}(1-x-u)^{\gamma-1} N(n, 1-u, t) d u \\
& =O(\varepsilon(n)) \int_{0}^{1-x}(1-x-u)^{\gamma-1} d u \\
& =O\left(\frac{\varepsilon(n)}{n^{r} t^{r}}\right),
\end{aligned}
$$


if $x>1-1 / n t$.

On the other hand if $x<1-1 / n t$, write

$$
\begin{aligned}
J_{1}= & \int_{0}^{1-x}(1-x-u)^{\gamma-1} N(n, 1-u, t) d u \\
= & \int_{0}^{1-x-1 / n t}(1-x-u)^{\gamma-1} N(n, 1-u, t) d u \\
& +\int_{1-x-1 / n t}^{1-x}(1-x-u)^{\gamma-1} N(n, 1-u, t) d u \\
= & J_{1,1}+J_{1,2},
\end{aligned}
$$

say. Since $\gamma<1$ and $(1-x-u)^{r-1}$ is an increasing function of $u$,

$$
\begin{aligned}
J_{1,1} & =\frac{1}{(n t)^{r-1}} \int_{\eta}^{1-x-1 / n t} N(n, 1-u, t) d u, \quad\left(0 \leqq \eta \leqq 1-x-\frac{1}{n t}\right) \\
& =O\left(\frac{\varepsilon(n)}{n^{\gamma} t^{\gamma}}\right)
\end{aligned}
$$

by the application of the estimate $I_{1}$ of Lemma 3 .

And using the estimate (4.1) we have

$$
J_{1,2}=O(\varepsilon(n)) \int_{1-x-1 / n t}^{1-x}(1-x-u)^{\gamma-1} d u=O\left(\frac{\varepsilon(n)}{n^{\gamma} t^{\gamma}}\right) .
$$

A combination of the estimates in (4.4) and (4.5), in view of (4.3), yields

$$
J_{1}=O\left(\frac{\varepsilon(n)}{n^{\gamma} t^{r}}\right)
$$

when $x<1-1 / n t$. Hence, in view of the estimates in (4.2) and (4.6), the first part of the lemma follows. The second part follows on similar lines.

5. Proof of the theorem. In view of the definition and Lemma 1, the absolute Hausdorff summability of the series $\sum \varepsilon(n) A_{n}(x)$ is equivalent to the absolute convergence of the series $\sum_{n=1}^{\infty} b_{n}$, where

$$
b_{n}=\frac{1}{n} \sum_{\nu=1}^{n} \nu\left(\begin{array}{l}
n \\
\nu
\end{array}\right)\left(\Delta^{n-\nu} \mu_{\nu}\right) \varepsilon(\nu) A_{\nu}(x) .
$$

We first consider the case $\alpha=0$.

Since

$$
\begin{aligned}
A_{\nu}(x) & =\frac{2}{\pi} \int_{0}^{\pi} \phi(t) \cos \nu t d t \\
& =-\frac{2}{\pi} \int_{0}^{\pi} \frac{\sin \nu t}{\nu} d \phi(t),
\end{aligned}
$$


and the transformation $(H, \mu)$ is convergence preserving,

$$
\begin{aligned}
b_{n} & =-\frac{2}{n \pi} \int_{0}^{\pi} d \dot{\phi}(t) \int_{0}^{1}\left(\sum_{\nu=1}^{n}\left(\begin{array}{l}
n \\
\nu
\end{array}\right) \varepsilon(\nu) x^{\nu}(1-x)^{n-\nu} \sin \nu t\right) d \chi(x) \\
& =-\frac{2}{n \pi} \int_{0}^{\pi} d \phi(t) \int_{0}^{1} N(n, x, t) d \chi(x),
\end{aligned}
$$

and therefore

$$
\begin{aligned}
\sum_{n=1}^{\infty}\left|b_{n}\right| \leqq & \frac{2}{\pi} \int_{0}^{\pi}|d \phi(t)|\left[\sum_{n=1}^{[11 t]} \frac{1}{n}\left|\int_{0}^{1} N(n, x, t) d \chi(x)\right|\right. \\
& \left.+\sum_{n=[1 / t]+1}^{\infty} \frac{1}{n}\left|\int_{0}^{1} N(n, x, t) d \chi(x)\right|\right] .
\end{aligned}
$$

Since

$$
\int_{0}^{\pi} \varepsilon\left(\frac{k}{t}\right)|d \phi(t)|<C,
$$

it is clear that we have to show that uniformly in $0<t \leqq \pi$,

$$
\sum_{1} \equiv \sum_{n=1}^{1,1 t]} \frac{1}{n}\left|\int_{0}^{1} N(n, x, t) d \chi(x)\right|=O\left(\varepsilon\left(\frac{k}{t}\right)\right)
$$

and

$$
\sum_{2} \equiv \sum_{n=[1 / t]+1}^{\infty} \frac{1}{n}\left|\int_{0}^{1} N(n, x, t) d \chi(x)\right|=O\left(\varepsilon\left(\frac{k}{t}\right)\right)
$$

Clearly

$$
\begin{aligned}
|N(n, x, t)| & \leqq \sum_{\nu=1}^{n}\left(\begin{array}{l}
n \\
\nu
\end{array}\right) \varepsilon(\nu) x^{\nu}(1-x)^{n-\nu} \nu t \\
& \leqq n t \varepsilon(n) \sum_{\nu=1}^{n}\left(\begin{array}{l}
n \\
\nu
\end{array}\right) x^{\nu}(1-x)^{n-\nu} \\
& \leqq n t \varepsilon(n),
\end{aligned}
$$

and therefore

$$
\begin{aligned}
\sum_{1} & =\sum_{n=1}^{[1 / t]} \frac{1}{n}\left|\int_{0}^{1} N(n, x, t) d \chi(x)\right| \\
& \leqq \sum_{n=1}^{[11 t]} \frac{n t \varepsilon(n)}{n} \int_{0}^{1}|d \chi(x)| \\
& =O\left(\varepsilon\left(\frac{k}{t}\right)\right),
\end{aligned}
$$

the function $\chi(x)$ being of bounded variation in $(0,1)$. This completes the proof of the estimate in (5.1). We now proceed to establish (5.2). Putting 
we have

$$
\chi(x)=g_{1+\gamma}^{+}(x)+C
$$

$$
\begin{aligned}
\sum_{2} & \equiv \sum_{n=[1 / t]+1}^{\infty} \frac{1}{n}\left|\int_{0}^{1} N(n, x, t) d \chi(x)\right| \\
& =\sum_{n=[1 / t]+1}^{\infty} \frac{1}{n}\left|\int_{0}^{1} g_{r}^{+}(x) N(n, x, t) d x\right| \\
& =\sum_{n=[1 / t]+1}^{\infty} \frac{1}{n}\left|\int_{0}^{1} g(x) N_{r}^{-}(n, x, t) d x\right|
\end{aligned}
$$

(by the application of Lemma 2)

$$
\leqq \int_{0}^{1}|g(x)|\left(\sum_{n=[1 / t]+1}^{\infty} \frac{1}{n}\left|N_{\gamma}^{-}(n, x, t)\right|\right) d x,
$$

where $N_{i}^{-}(n, x, t)$ means the $\gamma$ th backward fractional integral of $N(n, x, t)$ regarded as a function of $x$. By the application of Lemma 4 we get

$$
\begin{aligned}
N_{\gamma}^{-}(n, x, t) & =\frac{1}{\Gamma(\gamma)} \int_{x}^{1}(u-x)^{\gamma-1} N(n, u, t) d u \\
& =O\left(\frac{\varepsilon(n)}{n^{\gamma} t^{\gamma}}\right),
\end{aligned}
$$

and therefore

$$
\begin{aligned}
\sum_{2} & =O(1) \sum_{n=[1 / t]+1}^{\infty} \frac{\varepsilon(n)}{n^{1+\gamma} t^{r}} \int_{0}^{1}|g(x)| d x \\
& =O(1) \sum_{n=[1 / t]+1}^{\infty} \frac{\varepsilon(n)}{n^{1+\gamma} t^{\gamma}} \\
& =O\left(\varepsilon\left(\frac{k}{t}\right)\right),
\end{aligned}
$$

and this completes the proof of (5.2). The proof of the estimate in (5.2) in the case when

$$
\chi(x)=g_{1+\gamma}^{-}(x)+C
$$

follows on similar lines.

We now consider the case $0<\alpha<1$. We have

$$
\begin{aligned}
b_{n} & =\frac{1}{n} \int_{0}^{\pi} \phi(t) \frac{d}{d t}\left(\frac{2}{\pi} \sum_{\nu=1}^{n}\left(\begin{array}{l}
n \\
\nu
\end{array}\right) \varepsilon(\nu) \sin \nu t \int_{0}^{1} x^{\nu}(1-x)^{n-\nu} d \chi(x)\right) d t \\
& =\frac{1}{n} \int_{0}^{\pi} \phi(t) \frac{d}{d t} L(\chi ; n, t) d t \\
& =\frac{1}{n} \int_{0}^{\pi} \frac{d}{d t} L(\chi ; n, t)\left(\frac{1}{\Gamma(1-\alpha)} \int_{0}^{t}(t-u)^{-\alpha} d \Phi_{\alpha}(u)\right) d t \\
& =\frac{1}{n \Gamma(1-\alpha)} \int_{0}^{\pi} d \Phi_{\alpha}(u) \int_{u}^{\pi}(t-u)^{-\alpha} \frac{d}{d t} L(\chi ; n, t) d t
\end{aligned}
$$




$$
\begin{aligned}
& =\frac{1}{n} \int_{0}^{\pi} I(\chi ; n, u) d \Phi_{\alpha}(u) \\
& =\frac{1}{n} \Phi_{\alpha}(\pi) I(\chi ; n, \pi)-\frac{1}{n} \Phi_{\alpha}(\pi) J(\chi ; n, \pi)+\frac{1}{n} \int_{0}^{\pi} J(\chi ; n, u) d \phi_{\alpha}(u),
\end{aligned}
$$

and therefore in order to prove the theorem we have to show that

$$
\begin{aligned}
& \sum_{n=1}^{\infty} n^{-1}|I(\chi ; n, \pi)|<C, \\
& \sum_{n=1}^{\infty} n^{-1}|J(\chi ; n, \pi)|<C
\end{aligned}
$$

and

$$
\sum_{n=1}^{\infty} n^{-1}|J(\chi ; n, u)|=O\left(\varepsilon\left(\frac{k}{u}\right)\right),
$$

uniformly in $0<u \leqq \pi$.

We show that all the above estimates are true ${ }_{4}^{\pi}$ with

$$
\chi(x)=g_{1+\gamma}^{+}(x)+C \text {. }
$$

The method of proof for $\chi(x)=g_{1+\gamma}^{-}(x)+C$ will be similar.

For sake of brevity we write $g^{+}$for $g_{1+\gamma}^{+}$. We have

$$
\begin{aligned}
I\left(g^{+} ; n, u\right)= & \frac{1}{\Gamma(1-\alpha)} \int_{u}^{u+n^{-1}}(t-u)^{-\alpha} \frac{d}{d t} L\left(g^{+} ; n, t\right) d t \\
& +\frac{1}{\Gamma(1-\alpha)} \int_{u+n^{-1}}^{\pi}(t-u)^{-\alpha} \frac{d}{d t} L\left(g^{+}, n, t\right) d t \\
= & I_{1}\left(g^{+} ; n, u\right)+I_{2}\left(g^{+} ; n, u\right),
\end{aligned}
$$

say. Now

$$
\begin{aligned}
I_{1}\left(g^{+} ; n, u\right)= & \frac{2}{\pi \Gamma(1-\alpha)} \int_{u}^{u+n-1}(t-u)^{-\alpha} d t \int_{0}^{1} g_{\gamma}^{+}(x) \\
& \times\left(\sum_{\nu=1}^{n} \nu\left(\begin{array}{l}
n \\
\nu
\end{array}\right) \varepsilon(\nu) x^{\nu}(1-x)^{n-\nu} \cos \nu t\right) d x \\
= & \frac{2}{\pi \Gamma(1-\alpha)} \int_{u}^{u+n^{-1}}(t-u)^{-\alpha} d t \int_{0}^{1} g_{\gamma}^{+}(x) M(n, x, t) d x \\
= & \frac{2}{\pi \Gamma(1-\alpha)} \int_{u}^{u+n^{-1}}(t-u)^{-\alpha} d t \int_{0}^{1} g(x) M_{\zeta}^{-}(n, x, t) d x^{3} \\
= & \frac{2}{\pi \Gamma(1-\alpha) \Gamma(\gamma)} \int_{u}^{u+n^{-1}}(t-u)^{-\alpha} d t \int_{0}^{1} g(x) d x
\end{aligned}
$$

${ }^{3} M_{\gamma}^{-}(n, x, t)$ is $\gamma$ th backward fractional integral of $M(n, x, t)$ regarded as a function of $x$. 


$$
\begin{aligned}
& \times \int_{0}^{1-x}(1-x-v)^{r-1} M(n, 1-v, t) d v \\
= & O\left(\frac{\varepsilon(n)}{n^{\gamma-1}}\right) \int_{u}^{u+n^{-1}} t^{-\gamma}(t-u)^{-\alpha} d t \int_{0}^{1}|g(x)| d x \\
= & O\left(\frac{\varepsilon(n)}{n^{r-\alpha} u^{\gamma}}\right),
\end{aligned}
$$

by the application of the estimate of $J_{2}$ in Lemma 4 and using the fact that $g(x)$ is Lebesgue integrable in $(0,1)$.

Also

$$
\begin{aligned}
I_{2}\left(g^{+} ; n, u\right) & =\frac{1}{\Gamma(1-\alpha)} \int_{u+n^{-1}}^{\pi}(t-u)^{-\alpha} \frac{d}{d t} L\left(g^{+} ; n, t\right) d t \\
& =\frac{n^{\alpha}}{\Gamma(1-\alpha)} \int_{u+n^{-1}}^{\zeta} \frac{d}{d t} L\left(g^{+} ; n, t\right) d t, \quad\left(u+n^{-1}<\zeta<\pi\right)
\end{aligned}
$$

where

$$
\begin{aligned}
L\left(g^{+} ; n, t\right) & =\frac{2}{\pi} \int_{0}^{1}\left(\sum_{\nu=1}^{n}\left(\begin{array}{l}
n \\
\nu
\end{array}\right) \varepsilon(\nu) x^{\nu}(1-x)^{n-\nu} \sin \nu t\right) g_{\gamma}^{+}(x) d x \\
& =\frac{2}{\pi} \int_{0}^{1} g_{\gamma}^{+}(x) N(n, x, t) d x \\
& =\frac{2}{\pi} \int_{0}^{1} g(x) N_{\gamma}^{-}(n, x, t) d x \\
& =\frac{2}{\pi \Gamma(\gamma)} \int_{0}^{1} g(x) d x \int_{0}^{1-x}(1-x-v)^{\gamma-1} N(n, 1-v, t) d v \\
& =O\left(\frac{\varepsilon(n)}{n^{\gamma} t^{\gamma}}\right) \int_{0}^{1}|g(x)| d x \\
& =O\left(\frac{\varepsilon(n)}{n^{\gamma} t^{r}}\right),
\end{aligned}
$$

by the application of the estimate of $J_{1}$ in Lemma 4 and the Lebesgue integrability of $g(x)$ in $(0,1)$. Hence

$$
I_{2}\left(g^{+} ; n, u\right)=O\left(\frac{\varepsilon(n)}{n^{\gamma-\alpha} u^{\gamma}}\right) .
$$

Thus we have shown that

$$
I\left(g^{+} ; n, u\right)=O\left(\frac{\varepsilon(n)}{n^{\gamma-\alpha} u^{\gamma}}\right) .
$$

Using this estimate we have

$$
\sum_{n=1}^{\infty} n^{-1}\left|I\left(g^{+} ; n, \pi\right)\right| \leqq C \sum_{n=1}^{\infty} \frac{\varepsilon(n)}{n^{1+\gamma-\alpha}}<C,
$$


and this completes the proof of (5.6).

If in particular we suppose that $\phi(t)=1$ for all $t$, in which case $\phi_{\alpha}(t)=1$ for all $t$ and $b_{n}=0$ for every $n$, we obtain from (5.5) and the estimate in (5.9)

$$
O=O\left(\frac{\varepsilon(n)}{n^{1+\gamma-\alpha}}\right)-\frac{J\left(g^{+} ; n, \pi\right)}{n}
$$

and therefore

$$
J\left(g^{+} ; n, \pi\right)=O\left(\frac{\varepsilon(n)}{n^{\gamma-\alpha}}\right)
$$

Hence

$$
\sum_{n=1}^{\infty} n^{-1}\left|J\left(g^{+} ; n, \pi\right)\right| \leqq C \sum_{n=1}^{\infty} \frac{\varepsilon(n)}{n^{1+\gamma-\alpha}}<C,
$$

and this establishes the estimate in (5.7). Now it remains to establish (5.8). We note that

$$
\begin{aligned}
I(\chi ; n, u)= & \frac{1}{\Gamma(1-\alpha)}\left(\int_{u}^{u+n^{-1}}+\int_{u+n^{-1}}^{\pi}\right)(t-u)^{-\alpha} \frac{d}{d t} L(\chi ; n, t) d t \\
= & O(n \varepsilon(n)) \int_{u}^{u+n^{-1}}(t-u)^{-\alpha} d t \int_{0}^{1}\left(\sum_{\nu=1}^{n}\left(\begin{array}{c}
n \\
\nu
\end{array}\right) x^{\nu}(1-x)^{n-\nu}\right)|d \chi(x)| \\
& +\frac{2}{\pi} \cdot \frac{n^{\alpha}}{\Gamma(1-\alpha)} \sum_{\nu=1}^{n} \nu\left(\begin{array}{l}
n \\
\nu
\end{array}\right) \varepsilon(\nu) \int_{0}^{1} x^{\nu}(1-x)^{n-\nu} d \chi(x) \\
& \times \int_{u+n^{-1}}^{\zeta} \cos \nu t d t \\
= & O\left(n^{\alpha} \varepsilon(n)\right)+O\left(n^{\alpha} \varepsilon(n)\right) \int_{0}^{1}|d \chi(x)| \sum_{\nu=1}^{n}\left(\begin{array}{l}
n \\
\nu
\end{array}\right) x^{\nu}(1-x)^{n-\nu} \\
= & O\left(n^{\alpha} \varepsilon(n)\right),
\end{aligned}
$$

and therefore

$$
\begin{aligned}
J(\chi ; n, u) & =\frac{1}{\Gamma(1+\alpha)}\left[v^{\alpha} I(\chi ; n, v)\right]_{0}^{u}-\frac{\alpha}{\Gamma(1+\alpha)} \int_{0}^{u} v^{\alpha-1} I(\chi ; n, v) d v \\
& =O\left(n^{\alpha} u^{\alpha} \varepsilon(n)\right)+O\left(n^{\alpha} \varepsilon(n)\right) \int_{0}^{u} v^{\alpha-1} d v \\
& =O\left(n^{\alpha} u^{\alpha} \varepsilon(n)\right) .
\end{aligned}
$$

Also

$$
\Gamma(\alpha+1)[J(\chi ; n, \pi)-J(\chi ; n, u)]=\left[v^{\alpha} I(\chi ; n, v]_{u}^{\pi}-\alpha \int_{u}^{\pi} v^{\alpha-1} I(\chi ; n, v) d v\right.
$$

and therefore 


$$
\begin{aligned}
J\left(g^{+} ; n, u\right)= & J\left(g^{+} ; n, \pi\right)-\frac{1}{\Gamma(1+\alpha)}\left[v^{\alpha} I\left(g^{+} ; n, v\right]_{u}^{\pi}\right. \\
& +\frac{\alpha}{\Gamma(\alpha+1)} \int_{u}^{\pi} v^{\alpha-1} I\left(g^{+} ; n, v\right) d v \\
= & O\left(\frac{\varepsilon(n)}{n^{\gamma-\alpha}}\right)+O\left(\frac{\varepsilon(n)}{n^{\gamma-\alpha} u^{\gamma-\alpha}}\right)+O\left(\frac{\varepsilon(n)}{n^{\gamma-\alpha}}\right) \int_{u}^{\pi} v^{-\gamma+\alpha-1} d v \\
= & O\left(\frac{\varepsilon(n)}{n^{\gamma-\alpha} u^{\gamma-\alpha}}\right)
\end{aligned}
$$

using the estimates in (5.9) and (5.10). Now by the application of the estimate in (5.11) and (5.12) we have

$$
\begin{aligned}
\sum_{n=1}^{\infty} n^{-1}\left[J\left(g^{+} ; n, u\right) \mid\right. & =\sum_{n=1}^{[1 / u]} n^{-1}\left|J\left(g^{+} ; n, u\right)\right|+\sum_{n=[1 / u]+1}^{\infty} n^{-1}\left|J\left(g^{+} ; n, u\right)\right| \\
& =O\left(u^{\alpha}\right) \sum_{n=1}^{[11 u]} n^{\alpha-1} \varepsilon(n)+O(1) \sum_{n=[1 / u]+1}^{\infty} \frac{\varepsilon(n)}{n^{1+\gamma-\alpha} u^{\gamma-\alpha}} \\
& =O\left(\varepsilon\left(\frac{k}{u}\right)\right),
\end{aligned}
$$

uniformly in $0<u \leqq \pi$. This completes the proof of the estimate in (5.8). Hence the theorem.

We are thankful to the referee for his kind advice.

\section{REFERENCES}

1. L. S. Bosanquet, Note on the absolute summability (C) of a Fourier series, J. London Math. Soc., 11 (1936), 11-15.

2. - The absolute Cesàro summability of a Fourier series, Proc. London Math. Soc., (2), 41 (1936), 517-526,

3. G. H. Hardy, Divergent Series, (Oxford 1949).

4. K. Knoppand and G. G. Lorentz, Beitrage zur absoluten limitierung, Archiv der Math., 2 (1949-50), 10-16.

5. B. Kuttner, On the 'Second theorem of consistency' for Riesz summability (II), J. London Math. Soc., 27 (1952), 207-217.

6. R. Mohanty, The absolute Cesàro summability of some series associated with a Fourier series and its allied series, J. London Math. Soc., 25 (1950), 63-67.

7. — Absolute Cesàro summability of a series associated with a Fourier series, Bull. Calcutta Math. Soc., 44 (1952), 152-154.

8. H. Morley, A theorem on Hausdorff transformation and its application to Cesàro and Hölder means, J. London Math. Soc., 25 (1950), 168-173.

9. M. S. Ramanujan, On Hausdorff and quasi-Hausdorff methods of summability, Quart. J. Math., (Oxford) 8 (1957), 197-213.

10. N. Tripathi, On the absolute Hausdorff summability of some series associated with a Fourier series and its allied series, J. Indian Math. Soc., (New series) 32 (1968), 141154.

11. On the absolute Hausdorff summability of Fourier series, J. London Math. Soc., 44 (1969), 15-25. 
ON THE ABSOLUTE HAUSDORFF SUMMABILITY OF A FOURIER SERIES 451

12. D. V. Widder, Laplace transform (Princeton University Press, 1946)

Received January 13, 1971.

BANARAS HiNDU UNIVERSITY 



\section{PACIFIC JOURNAL OF MATHEMATICS}

\section{EDITORS}

\author{
H. SAMELSON \\ Stanford University \\ Stanford, California 94305 \\ C. R. HOBBY \\ University of Washington \\ Seattle, Washington 98105
}

\section{J. DuGundJI}

Department of Mathematics University of Southern California Los Angeles, California 90007

RICHARD ARENS

University of California

Los Angeles, California 90024

\section{ASSOCIATE EDITORS}
E. F. BECKENBACH
B. H. NeumanN
F. WOLF
K. YOSHIDA

\section{SUPPORTING INSTITUTIONS}

\author{
UNIVERSITY OF BRITISH COLUMBIA \\ CALIFORNIA INSTITUTE OF TECHNOLOGY \\ UNIVERSITY OF CALIFORNIA \\ MONTANA STATE UNIVERSITY \\ UNIVERSITY OF NEVADA \\ NEW MEXICO STATE UNIVERSITY \\ OREGON STATE UNIVERSITY \\ UNIVERSITY OF OREGON \\ OSAKA UNIVERSITY
}

\author{
UNIVERSITY OF SOUTHERN CALIFORNIA \\ STANFORD UNIVERSITY \\ UNIVERSITY OF TOKYO \\ UNIVERSITY OF UTAH \\ WASHINGTON STATE UNIVERSITY \\ UNIVERSITY OF WASHINGTON \\ AMERICAN MATHEMATICAL SOCIETY \\ NAVAL WEAPONS CENTER
}

The Supporting Institutions listed above contribute to the cost of publication of this Journal, but they are not owners or publishers and have no responsibility for its content or policies.

Mathematical papers intended for publication in the Pacific Journal of Mathematics should be in typed form or offset-reproduced, (not dittoed), double spaced with large margins. Underline Greek letters in red, German in green, and script in blue. The first paragraph or two must be capable of being used separately as a synopsis of the entire paper. The editorial "we" must not be used in the synopsis, and items of the bibliography should not be cited there unless absolutely necessary, in which case they must be identified by author and Journal, rather than by item number. Manuscripts, in duplicate if possible, may be sent to any one of the four editors. Please classify according to the scheme of Math. Rev. Index to Vol. 39. All other communications to the editors should be addressed to the managing editor, Richard Arens, University of California, Los Angeles, California, 90024.

50 reprints are provided free for each article; additional copies may be obtained at cost in multiples of 50 .

The Pacific Journal of Mathematics is published monthly. Effective with Volume 16 the price per volume (3 numbers) is $\$ 8.00$; single issues, $\$ 3.00$. Special price for current issues to individual faculty members of supporting institutions and to individual members of the American Mathematical Society: $\$ 4.00$ per volume; single issues $\$ 1.50$. Back numbers are available.

Subscriptions, orders for back numbers, and changes of address should be sent to Pacific Journal of Mathematics, 103 Highland Boulevard, Berkeley, California, 94708.

PUBLISHED BY PACIFIC JOURNAL OF MATHEMATICS, A NON-PROFIT CORPORATION

Printed at Kokusai Bunken Insatsusha (International Academic Printing Co., Ltd.), 270, 3-chome Totsuka-cho, Shinjuku-ku, Tokyo 160, Japan. 


\section{Pacific Journal of Mathematics}

\section{Vol. 42, No. $2 \quad$ February, 1972}

Stephen Richard Bernfeld, The extendability of solutions of perturbed scalar differential equations ................................. 277

James Edwin Brink, Inequalities involving $f_{-} p$ and $f^{(n)}{ }_{q}$ for $f$ with $n$

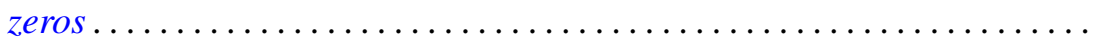

Orrin Frink and Robert S. Smith, On the distributivity of the lattice of filters of a groupoid

Donald Goldsmith, On the density of certain cohesive basic sequences .... 323

Charles Lemuel Hagopian, Planar images of decomposable continua . . . . . 329

W. N. Hudson, A decomposition theorem for biadditive processes ........ 333

W. N. Hudson, Continuity of sample functions of biadditive processes......

Masako Izumi and Shin-ichi Izumi, Integrability of trigonometric series.

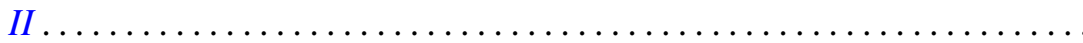

H. M. Ko, Fixed point theorems for point-to-set mappings and the set of

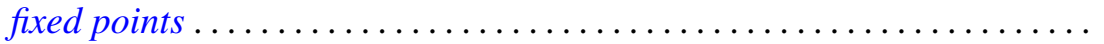

Gregers Louis Krabbe, An algebra of generalized functions on an open interval: two-sided operational calculus ...

Thomas Latimer Kriete, III, Complete non-selfadjointness of almost selfadjoint operators.................................

Shiva Narain Lal and Siya Ram, On the absolute Hausdorff summability of a Fourier series .

Ronald Leslie Lipsman, Representation theory of almost connected groups...

James R. McLaughlin, Integrated orthonormal series.... . .

H. Minc, On permanents of circulants.

Akihiro Okuyama, On a generalization of $\Sigma$-spaces.....

Norberto Salinas, Invariant subspaces and operators of class $(S)$

James D. Stafney, The spectrum of certain lower triangular matrices as operators on the $l_{p}$ spaces .......................

Arne Stray, Interpolation by analytic functions

$\mathrm{Li} \mathrm{Pi} \mathrm{Su}$, Rings of analytic functions on any subset of the complex plane.

R. J. Tondra, A property of manifolds compactly equivalent to compact manifolds.... 